\title{
Spectrum of electron-hole pair in quantum dots of a semiconductor nanolaser: theory
}

\author{
S.I. Pokutnyi \\ Illichivsk Educational Research Center of the Odessa National University: \\ 17 A Danchenko str., Illichivsk, Odessa red., 68001, Ukraine; \\ E-mail: univer@ivt.ilyichevsk.odessa.ua, Phone/fax: + 380 (4868) 43076
}

\begin{abstract}
A theory of laser generation on size-quantization levels in semiconductor quantum dots put in a semiconductor matrix is developed. The size of quantum dots mass of which comprises the active sphere of an injection laser is determined by the new optical method consisting of the comparison of theoretical and experimental dependence of the spectrum of an electron-hole pair upon the radius of a quantum dot.
\end{abstract}

Keywords: energy spectrum, electron-hole pair, quantum dots; semiconductor nanolaser.

Paper received 26.03.04; accepted for publication 21.10.04.

\section{Introduction}

Quasi-zero-dimensional structures consisting of spherical semiconductor microcrystals with a radius of $a \sim 1-10^{2} \mathrm{~nm}$ (the so-called quantum dots (QDs)), grown in transparent semiconductor (dielectric) matrices [1-12], draw attention due to their non-linear optical properties and applications in optoelectronics (in particular, as an active range of injection semiconductor lasers [10, 13-17]).

Optical and non-linear optical properties of such multiphase systems are determined by the energy spectrum of space-limited electron-hole pairs (excitons) [112, 18-21]. The energy spectrum of charge carriers in QD of sizes $a \sim 1-10^{2} \mathrm{~nm}$ will be absolutely discrete [22, 23]. This property of QD is used for creating optical nanolasers and other devices with high temperature stability of frequency generation $[10,14,15]$. The QDs dimensions a must be in the range of several $\mathrm{nm}$ for appearing energy gaps between the levels of electrons and holes $E_{e(h)}$ to be of the order of several $k T_{0}\left(\Delta E_{e(h)} \sim k T_{0}\right.$, where $k$ is the Bolzmann constant) and $T_{0}$ is the room temperature.

In experimental paper [14], optical properties of vertically coupled InAs QDs in GaAs matrix and connected with them instrumental characteristics of injection laser with an active range in terms of the mass QD were studied. A strong short-wave shift of the laser generation line of the mass QD was observed at that time.
In this present paper, the theory of size-quantization of laser generation levels in semiconductor QDs placed into a semiconductor matrix is developed. The expression for the Hamiltonian of an electron-hole pair in QD, which contains both the energy of the Coulomb interaction between the electron and hole as well as the energy of interaction between electrons and holes with the field of induced polarization on a spherical interface between two media has been obtained.

The value of the quantum dots mass of which comprises the active sphere of the injection laser is determined by the new optical method [19-23] consisting of comparison of theoretical and experimental $[14,15]$ dependence of the spectrum of electron-hole pair on QD radius $a$.

\section{Hamiltonian of an electron-hole pair in a quantum dot}

We shall consider a simple model of a quasi-zero-dimensional system: of a neutral spherical semiconductor QD of radius $a$ with a dielectric function $\varepsilon_{2}$ surrounded by a medium dielectric function of which is $\varepsilon_{1}$. An electron $e$ and a hole $h$ with the effective masses $m_{e}$ and $m_{h}$, respectively, move in this QD $\left(r_{e}\right.$ and $r_{h}$ are the distances of the electron and hole from the center of QD). It is also assumed that the electron and hole energy bands are parabolic. 


\section{S.I. Pokutnyi: Spectrum of electron-hole pair in quantum dots of ...}

The characteristic dimensions of the problem are $a$, $a_{e}, a_{h}, a_{e x}$, where

$a_{e}=\varepsilon_{2} \hbar^{2} / m_{e} e^{2}, a_{h}=\varepsilon_{2} \hbar^{2} / m_{h} e^{2}$,

$a_{e x}=\varepsilon_{2} \hbar^{2} / \mu e^{2}$,

are the Bohr radii of an electron, a hole and an exciton in a semiconductor with the dielectric function $\varepsilon_{2}(e$ is the electron charge, $\mu=m_{e} m_{h} /\left(m_{e}+m_{h}\right)$ is the reduced effective mass of the exciton). The fact that all the characteristic dimensions of the problem

$a, a_{e}, a_{h}, a_{e x} \gg a_{0}$

are much greater than the interatomic distance $a_{0}{ }^{1}$, allows us to consider the motion of an electron and hole in QD in the effective mass approximation.

The conditions of charge-carrier localization near a spherical dielectric particle were analyzed in Ref. [18, 23, 25], where the problem concerning the field induced by the charge near the dielectric particle, put in a dielectric medium, is solved and the analytic expressions for potential energy of interaction of charge carriers with the spherical interface between these two media are given.

In particular, in Refs $[18,23,25]$ shown is that the potential at the observation point $r^{\prime}$ in the medium $\varepsilon_{j}$ induced by a charge $e$ at the point $r$ in the medium $\varepsilon_{i}$ can be represented as the sum of potentials provided by the point charge of the image $e^{\prime}\left(r_{i j} / r\right)$ at the point $\left(r_{i j}=(a / r)^{2} r \delta_{i j}+\right.$ $\left.+r\left(1-\delta_{i j}\right)\right)$ and the linear distribution, with the density $\rho_{i j}(y, r)$, of the image charge along the straight line which goes through the sphere center and the charge at the point $r$ :

$$
\begin{aligned}
& \varphi\left(r^{\prime}, j / r, i\right)=\left(\frac{e}{\varepsilon_{j}\left(r^{\prime}-r\right) 0}\right)+\left(\frac{e^{\prime}\left(r_{i j} / r\right)}{\varepsilon_{j}\left(r^{\prime}-r_{i j}\right)}\right)+ \\
& +\frac{1}{\varepsilon_{j}} \int_{0}^{\infty} \frac{d y \rho_{i j}(y, r)}{\left(r^{\prime}-y\left(r / r^{\prime}\right)\right)} .
\end{aligned}
$$

The first term in (3) determines the field, created by the charge.

In case, when the charge $e$ is situated in the point $r$ in the bulk QD with $\varepsilon_{2}$, and the observation point $r^{\prime}$ is also situated in the bulk QD $(i=j=2)$, quantities $\varepsilon^{\prime}\left(r_{22} \mid r\right), r_{22}$ and $\rho_{22}(y, r)$ are determined in the following way:

$$
\begin{aligned}
& e^{\prime}\left(r_{22} / r\right)=\frac{a}{r} \beta e, r_{22}=\left(\frac{a^{2}}{r^{2}}\right), \\
& \rho_{22}(y, r)=\beta(1-\alpha)\left(\frac{a^{2}}{r y}\right)^{\alpha} \frac{e}{a} \Theta\left(y-\frac{a^{2}}{r}\right) \\
& \beta=\frac{\varepsilon_{2}-\varepsilon_{1}}{\varepsilon_{2}+\varepsilon_{1}}, \alpha=\frac{\varepsilon_{1}}{\varepsilon_{2}+\varepsilon_{1}}
\end{aligned}
$$

where $\Theta(x)$ is the conventional step function.
Using the adopted model of quasi-zero-dimensional system, we can write down the potential energy of interaction of the electron $e$ and hole $h$ (with the charge (-e)), in the bulk QD at the points $r_{e}$ and $r_{h}$, with the field induced by these quasiparticles polarization in the following way:

$V\left(r_{e}, r_{h}, a\right)=\frac{e}{2} \varphi\left(r_{e}, 2 \mid r_{h}, 2\right)-\frac{e}{2} \varphi\left(r_{h}, 2 \mid r_{e}, 2\right)$

where potentials $\varphi\left(r_{e}, 2 \mid r_{h}, 2\right)$ and $\varphi\left(r_{h}, 2 \mid r_{e}, 2\right)$ created by the hole being at the point $r_{h}$, at the point $r_{e}$ of the position of the electron and by the electron being at the point re, at the point rh of the position of the hole due to Eqs (3) and (4) becomes:

$$
\varphi\left(r_{e}, 2 \mid r_{h}, 2\right)=-\frac{e}{\varepsilon_{2}\left(r_{h}-r_{e}\right)}-
$$

$$
\begin{aligned}
& -\frac{\beta e}{\varepsilon_{2} a}\left(\frac{1}{\left[\left(r_{e} r_{h} / a^{2}\right)^{2}-2\left(r_{e} r_{h} / a^{2}\right) \cos \theta+1\right]^{1 / 2}}\right)- \\
& -\frac{\beta}{\varepsilon_{2}+\varepsilon_{1}} \frac{e}{a} \int_{0}^{\infty} \frac{d y\left(a^{2} / r_{h} y\right)^{a} \Theta\left(y-\left(a^{2} / r_{h}\right)\right)}{r_{e}-y\left(r_{h} / r_{h}\right)},
\end{aligned}
$$

$$
\varphi\left(r_{h}, 2 \mid r_{e}, 2\right)=-\frac{e}{\varepsilon_{2}\left(r_{h}-r_{e}\right)}-
$$

$$
\begin{aligned}
& -\frac{\beta e}{\varepsilon_{2} a}\left(\frac{1}{\left[\left(r_{e} r_{h} / a^{2}\right)^{2}-2\left(r_{e} r_{h} / a^{2}\right) \cos \theta+1\right]^{1 / 2}}\right)- \\
& -\frac{\beta}{\varepsilon_{2}+\varepsilon_{1}} \frac{e}{a} \int_{0}^{\infty} \frac{d y\left(a^{2} / r_{e} y\right)^{a} \Theta\left(y-\left(a^{2} / r_{e}\right)\right)}{r_{h}-y\left(r_{e} / r_{e}\right)}
\end{aligned}
$$

where the corner $\Theta=r_{e} r_{h}$.

Using Eqs (6), the energy potential interaction $V\left(r_{e}\right.$, $\left.r_{h}, a\right)(5)$, becomes:

$V\left(r_{e}, r_{h}, a\right)=V_{e h}\left(r_{e}, r_{h}\right)+U\left(r_{e}, r_{h}, a\right)$,

where

$$
V_{e h}\left(r_{e}, r_{h}\right)=-\frac{e^{2}}{\varepsilon_{2}\left|r_{h}-r_{e}\right|}-
$$

is the energy of Coulomb interaction being an electron and a hole, and

$$
\begin{aligned}
& U\left(r_{e}, r_{h}, a\right)=-\frac{e^{2} \beta}{\varepsilon_{2} a}\left(\frac{1}{\left[\left(r_{e} r_{h} / a^{2}\right)^{2}-2\left(r_{e} r_{h} / a^{2}\right) \cos \theta+1\right]^{1 / 2}}\right)- \\
& -\frac{e^{2} \beta}{2\left(\varepsilon_{2}+\varepsilon_{1}\right) a} \int_{0}^{\infty} \frac{d y\left(a^{2} / r_{h} y\right)^{a} \Theta\left(y-\left(a^{2} / r_{h}\right)\right)}{r_{e}-y\left(r_{h} / r_{h}\right)}- \\
& -\frac{e^{2} \beta}{2\left(\varepsilon_{2}+\varepsilon_{1}\right) a} \int_{0}^{\infty} \frac{d y\left(a^{2} / r_{e} y\right)^{a} \Theta\left(y-\left(a^{2} / r_{e}\right)\right)}{r_{h}-y\left(r_{e} / r_{e}\right)} .
\end{aligned}
$$

Is the energy of interaction of an electron and a hole with the induced polarization field at the spherical interface between these two media. 


\section{S.I. Pokutnyi: Spectrum of electron-hole pair in quantum dots of ...}

The Hamiltonian of an exciton obtained subject to the above assumption has the following form in the adopted model of this quasi-zero-dimensional system

$$
\begin{aligned}
& H=-\frac{\hbar^{2}}{2 m_{e}} \Delta_{e}-\frac{\hbar^{2}}{2 m_{h}} \Delta_{h}+ \\
& +V_{e h}\left(r_{e}, r_{h}\right)+U\left(r_{e}, r_{h}, a\right)+E_{g},
\end{aligned}
$$

where the first two terms describe the kinetic energy of an electron and a hole, $E_{g}$ is the band gap in an infinite semiconductor with the dielectric function $\varepsilon_{2}$, and energy potential interaction $V_{e h}\left(r_{e}, r_{h}\right), U\left(r_{e}, r_{h}, a\right)$ are described by Eqs (8) and (9).

\section{Short-wave length shift of the line of laser generation in the semiconductor quantum dots}

Let's consider the results of the experiment [14]. In this paper, optical properties of the masses of vertically aligned pyramidal QD of GaAs (with the dielectric function $\left.\varepsilon_{2}=12,5\right)$ with an average dimension $a \cong 5 \mathrm{~nm}$ in the matrix $\operatorname{GaAs}\left(\varepsilon_{1}=12\right)$ are studied. By an average dimension of $a$ of this QD we mean an average radius a between the sphere described around QD and the sphere inscribed in the bulk of QD. A strong short-wave length shift of the line of laser generation $\Delta E=106 \mathrm{meV}$ of the mass QD by the temperature $T=77 \mathrm{~K}$ was observed.

The mass QD under study in [14] was fired by the $T=$ $=973 \mathrm{~K}$ during the period not exceeding $60 \mathrm{~min}$. Shortwave shift of the laser generation line the authors [14] related with the reduction in the energy of localization of charge carriers in the volume QD which was caused by their annealing.

Since in this [14] quazi-zero-dimensional structure the dielectric function of QD and the surrounding matrix are assumed to differ slightly (the parameter $\beta=2 \cdot 10^{-2}$ (4)), in the Hamiltonian (10) in a first approximation the quantity of the energy of the polarization interaction of an electron and a hole $U\left(r_{e}, r_{h}, a\right)(9)$ may be neglected. As a result, the Hamiltonian (10) becomes:

$$
H=-\frac{\hbar^{2}}{2 m_{e}} \Delta_{e}-\frac{\hbar^{2}}{2 m_{h}} \Delta_{h}+V_{e h}\left(r_{e}, r_{h}\right)+E_{g},
$$

Effective masses of an electron and a hole in QD of GaAs had respectively equals $\left(m_{e} / m_{0}\right)=2.8 \cdot 10^{-2}$ and $\left(m_{h} / m_{0}\right)=3.3 \cdot 10^{-1}[26]\left(m_{e} / m_{h}\right)=8.5 \cdot 10^{-2}$, i.e. $\left.m_{e}<<m_{h}\right)$.

It's assumed that as in $[27,28]$ during the annealing of the volume QD by the $T=973 \mathrm{~K}$ heat ejection of a light-weight electron occurs and the bulk of QD contains only a hole. In the Hamiltonian (11), we shall neglect the energy of the Coulomb interaction $V_{e h}(8)$ of the hole with the electron that can be localized at a deep trap in the matrix of GaAs. This is justified provided the distance $\mathrm{d}$ between the trap and the center of the QD is large as compared with the radius of QD a, i.e. if $d>>a$.
As a result, the effect of the spherical boundary QD of the radius a on the spectrum of the hole $E_{n l}(a)(n, l-$ the principal and orbital quantum numbers) cause the shift of all levels of size quantization of the hole:

$\Delta E_{n l}(a) \frac{\hbar^{2}}{2 m_{h} a^{2}} \varphi_{n l}^{2}$

where $\Delta \varphi_{n l}$ are the roots of the equation for Bessel functions $J_{1+1 / 2}\left(\varphi_{n l}\right)=0$. Eq. (12) was obtained assuming that the minimal value of hole energy is located at the center of QD Brillouin zone, the top of the valence band is taken as a zero.

QD dispersion to dimensions being neglected, let's assume that short-wave shift of the line of laser generation $\Delta E=106 \mathrm{meV}$ of the mass $\mathrm{QD}$, which is due to the size quantization of the hole, can be written as follows (12):

$$
\Delta E=\frac{\hbar^{2}}{2 m_{h} a^{2}} \varphi_{10}^{2}
$$

where $\varphi_{10}=\pi$. Eq. (13) allows to determine an average dimension of QD $a \sim 3.3 \mathrm{~nm}$ studied under the conditions of the experiments [14].

\section{Conclusions}

The Hamiltonian of an electron-hole pair (11) in QD of GaAs and quantities $\Delta E(12),(13)$ were obtained assuming that electron and hole bands are parabolic. Moreover, Eq. (12), (13) were found assuming that QD of GaAs is spherical, symmetric potential well of an infinite depth for the hole. Such approximations are justified only for the lowest level of the hole $(n, l)$ in QD for which the condition can be satisfied $\Delta E_{n, l}(a)<<V_{0}$ (where $V_{0}$ is the depth of the potential well for holes in QD).

Thus, using the new optical method based on the comparison of theoretical and experimental dependencies of the energy spectrum $\Delta E_{n, l}(a)$ of charge carriers on QD radius a gives the possibility to determine the size of quantum dot, mass of which comprises the active sphere of injection laser.

\section{References}

1. V.G. Litovchenko, Osnovy fiziki poluprovodnikovykh sloistykh system, (Naukova Dumka, Kiev, 1980).

2. V.M. Agranovich, Yu.E. Lozovik // JETP Lett., 17(4), p. 209 (1973).

3. M.F. Deygen, M.D. Glinchuk // Sov. Phys. Sol. Stat., 5(11), p. 3250 (1963).

4. V.G. Litovchenko // Thin Sol. Films, 36(1), p. 205 (1976).

5. V.A. Zuev, D.V. Korbutyak, M.V. Kurik, V.G. Litovchenko // JETP Lett., 26(6) p. 455 (1977).

6. E.P. Pokatilov, S.I. Beril, V.M. Fomin, V.G. Litovchenko, D.V. Korbutyak // Phys. Stat. Sol. (b), 145(2), p. 535 (1988).

7. M.R. Kulish, V.P. Kunets, M.P. Lisitsa // Sb.: Kvantovaya elektronika, 46, p. 75 (1994).

8. M.R. Kulish, V.P. Kunets, M.P. Lisitsa // Ukr. Fiz. Zh., 35(12), p. 1817 (1990). 


\section{S.I. Pokutnyi: Spectrum of electron-hole pair in quantum dots of ...}

9. A.D. Yoffe // Advanc. Phys., 42, p. 173 (1993).

10. J.I. Alferov // Fiz. Tekh. Poluprovodn., 32, p. 3 (1998).

11. M.R. Kulish, V.P. Kunets, M.P. Lisitsa // Ukr. Fiz. Zh., 41(11, 12), 1865 (1996).

12. M.R. Kulish, V.P. Kunets, M.P. Lisitsa // Phys. Sol. Stat., 39(10), 1865 (1997).

13. A.I. Ekimov, A.A. Onushchenko // JETP Lett., 40, p. 1136 (1984).

14. A.E. Zhukov, A.Ju. Egorov, A.R. Kovsh // Fiz. Tekh. Poluprovodn., 31, p. 104 (1997).

15. V.P. Evtikhiev, I.V. Kudryashov, E.Ju. Kotelnikov // Fiz. Tekh. Poluprovodn., 32, p. 1482 (1998).

16. A.F. Tzatzulnikov, N.N. Ledentzov, M.V. Maksimov // Fiz. Tekh. Poluprovodn., 30, p. 1793 (1996).

17. A.F. Tzatzulnikov, N.N. Ledentzov, M.V. Maksimov // Fiz. Tekh. Poluprovodn., 30, p. 1822 (1996).

18. N.A. Efremov, S.I. Pokutnii // Fiz. Tverd. Tela, 27, (1985) 48 [Sov. Phys. Solid State 27, 27 (1985)].

19. S.I. Pokutnii // Phys. Lett. A., 203, p. 388 (1995).

20. S.I. Pokutnii // Fiz. Tverd. Tela, 38, p. 512 (1996); 38, p. 2667 (1996) [Phys. Solid State 38, p. 281 (1996); 38, p. 1463 (1996)].
21. S.I. Pokutnii // Fiz. Tekh. Poluprovodn., 30, p. 1320 (1996); 30, p. 1952 (1996) [Semiconductors, 30, p. 694 (1996); 30 p. 1015 (1996)].

22. S.I. Pokutnii // Fiz. Tekh. Poluprovodn., 31, p. 1447 (1997) [Semiconductors, 31, p. 1247 (1997)].

23. S.I. Pokutnii // Phys. Stat. Sol. (b), 165, p. 109 (1991); 172, p. 573 (1992); Fiz. Tverd. Tela, 35, p. 257 (1993) [Phys. Solid State, 35, p. 129 (1993)].

24. Yu.E. Losovik, D.R. Musin, Preprint №20, (Inst. Spektroskopy Akad. Nauk USSR, 1981).

25. N.A. Efremov, S.I. Pokutnii, Preprint №1, (Inst. Spektroskopy Akad. Nauk USSR, 1984).

26. V.I. Gavrilenko, A.M. Grekov, D.V. Korbutyak, V.G. Litovchenko Opticheskie svojstva poluprovodnikov: Handbook (Naukova Dumka, Kiev 1987).

27. V. Grabovskis, Ya. Dzenis, A. Ekimov // Fiz. Tverd. Tela, 31, p. 272 (1989) [Sov. Phys. Solid State, 31, p. 272 (1989)].

28. D. Bimberg, N.N. Ledentsov, N. Kirstaedter, O. Schmidt etc., Proc. 1995 Int. Conf. On Solid State Devices and Materials, (Osaka, 1995). 